\title{
Avaliação do Conhecimento dos Acadêmicos de Enfermagem em Genética/Genômica sobre o Câncer de Mama
}

doi: https://doi.org/10.32635/2176-9745.RBC.2021v67n3.1337

\author{
Assessment of the Knowledge of Nursing Academics in Genetics/Genomics about Breast Cancer \\ Evaluación del Conocimiento de Academias de Enfermería en Genética/Genómica sobre el Cáncer de Mama
}

Ingrid Renny Silva Palha'; Cintia Yolette Urbano Pauxis Aben-Athar ${ }^{2}$; Karolayne Teles Costa ${ }^{3}$; Renata Glaucia Barros da Silva Lopes ; Michele Monteiro Sousa ${ }^{5}$; Glenda Roberta Oliveira Naiff Ferreira ${ }^{6}$; Aline Maria Pereira Cruz Ramos ${ }^{7}$

RESUMO

Introdução: As universidades de enfermagem não têm acompanhado as demandas relativas às disciplinas de genética, genômica ou oncologia para aperfeiçoar o conhecimento dos acadêmicos no manejo do câncer de mama, que é o mais comum entre as mulheres brasileiras, exigindo maior eficácia das políticas de detecção precoce, tratamento oportuno e aconselhamento genético. Isso se deve em parte à não obrigatoriedade de oferecer essas disciplinas na grade curricular, o que pode levar a um déficit de conhecimento e possível prejuízo da futura qualidade desses profissionais. Objetivo: Analisar se o conhecimento dos acadêmicos de enfermagem sobre os conceitos de genética e genômica aplicados ao câncer de mama está associado à grade curricular das instituiçôes onde estudam. Método: Estudo multicêntrico, transversal, norteado pela ferramenta STROBE, realizado entre agosto/outubro de 2018. Resultados: Acadêmicos de instituições públicas apresentaram correlação entre a ausência das disciplinas genética/genômica ( $\mathrm{p}=0,0001)$ e pouco conhecimento dos respectivos conceitos $(\mathrm{p}=0,0045)$. Alternativamente, os de instituiçôes privadas mostraram maiores erros em relação ao exame clínico de mama anual a partir dos 40 anos $(\mathrm{p}=0,0009)$ e à periodicidade do rastreio mamográfico na população sob risco geral $(\mathrm{p}=0,0021)$. Os dois grupos convergiram na recomendação da mamografia à população sob risco familiar entre 35-69 anos. Conclusáo: Os acadêmicos das instituições de ensino superior privadas apresentaram maiores acertos sobre conceitos de genética/genômica, pois continham a disciplina genética na grade curricular, enquanto os das instituiçóes públicas se destacaram nos acertos relacionados ao câncer de mama sobre políticas de saúde, em razão da maior vivência prática no estágio curricular.

Palavras-chave: Genética/educação; Genômica/educação; Neoplasias da Mama; Estudantes; Pesquisa em Avaliação de Enfermagem.

\section{ABSTRACT}

Introduction: Nursing universities have not kept up with the demands related to the disciplines of genetics, genomics, or oncology to improve the knowledge of students in managing breast cancer, which is the most common among Brazilian women, demanding greater effectiveness of policies for early detection, timely treatment, and genetic counseling. This is partly due to the fact that it is not mandatory to offer these subjects in the curriculum, which can lead to a deficit of knowledge potentially harmful to the future quality of these professionals. Objective: To analyze whether the knowledge of nursing students about the concepts of genetics and genomics applied to breast cancer is associated with the curriculum of the institutions where they study. Method: Multicenter, cross-sectional study, guided by the STROBE tool, carried out between August-October 2018. Results: Students from public institutions showed correlation between the absence of genetics/genomics disciplines $(\mathrm{p}=0.0001)$ and poor knowledge of the respective concepts $(\mathrm{p}=0.0045)$. Alternatively, those from private institutions showed more errors in relation to the annual clinical breast exam from the age of $40(\mathrm{p}=0.0009)$ and the frequency of mammographic screening in the population at general risk $(\mathrm{p}=0.0021)$. The two groups concurred in recommending mammography to the population at risk between 35 and 69 years of age. Conclusion: Students from private universities where genetics is included in the disciplines were more cognizant about concepts of genetics and genomics, while those from public institutions stood out regarding correct responses on breast cancer related health policies because of their internship practice.

Key words: Genetics/education; Genomics/education; Breast Neoplasms; Students; Nursing Evaluation Research.

\section{RESUMEN}

Introducción: Las universidades de enfermería no se han mantenido al día con las demandas relacionadas con las disciplinas de genética, genómica u oncología para mejorar el conocimiento de los académicos en el manejo del cáncer de mama, que es el más común entre las mujeres brasileñas, exigiendo una mayor efectividad de las políticas de detección precoz. tratamiento oportuno $\mathrm{y}$ asesoramiento genético. Esto se debe en parte a que no es obligatorio ofrecer estas asignaturas en el plan de estudios, lo que puede conllevar un desconocimiento y posibles daños a la calidad futura de estos profesionales. Objetivo: Analizar si el conocimiento de los estudiantes de enfermería sobre los conceptos de genética y genómica aplicados al cáncer de mama está asociado al currículo de las instituciones donde cursan estudios. Método: Estudio multicéntrico, transversal, guiado por la herramienta STROBE, realizado entre agosto y octubre de 2018. Resultados: Los académicos de las instituciones públicas mostraron una correlación entre la ausencia de disciplinas de genética/ genómica ( $\mathrm{p}=0,0001)$ y el escaso conocimiento de los conceptos respectivos $(\mathrm{p}=0,0045)$. Alternativamente, las de instituciones privadas mostraron mayores errores en relación al examen clínico de mama anual a partir de los 40 ańos $(\mathrm{p}=0,0009)$ y la frecuencia de cribado mamográfico en la población de riesgo general $(\mathrm{p}=0,0021)$. Los dos grupos convergieron en la recomendación de la mamografía a la población de riesgo entre los 35 y los 69 ańos. Conclusión: Los académicos de las instituciones privadas de educación superior fueron más correctos sobre los conceptos de genética y genómica, ya que incluyeron la disciplina genética en el plan de estudios, mientras que los de las instituciones públicas se destacaron en las respuestas correctas relacionadas con el cáncer de mama en las políticas de salud, debido a la mayor experiencia práctica en la pasantía curricular.

Palabras clave: Genética/educación; Genómica/educación; Neoplasias de la Mama; Estudiantes; Investigación en Evaluación de Enfermería.

'Universidade de São Paulo (USP). Hospital das Clínicas da Faculdade de Medicina. São Paulo (SP), Brasil. E-mail: ingridrpalha@gmail.com. Orcid iD: https://orcid. org/0000-0002-3900-1495

2,67, Universidade Federal do Pará (UFPA). Faculdade de Enfermagem. Belém (PA), Brasil. E-mails: abenathar_cintia@hotmail.com; grnaiff@gmail.com; nurse.alinecruz@gmail.com. Orcid iD: https://orcid.org/0000-0002-6951-3547; Orcid iD: https://orcid.org/0000-0002-8206-4950; Orcid iD: https://orcid.org/0000-0001-8812-2923 ${ }_{3}^{3}$ Universidade da Amazônia (Unama). Belém (PA), Brasil. E-mail: costakarolyne1@gmail.com. Orcid iD: https://orcid.org/0000-0002-8036-6605 ${ }^{4}$ UFPA. Hospital Universitário João de Barros Barreto (HUJBB). Belém (PA), Brasil. E-mail: renatagbsilva@yahoo.com.br. Orcid iD: https://orcid.org/0000-0002-9232-5560 ${ }^{5}$ Secretaria de Estado de Saúde Pública do Estado do Pará (SESPA). Belém (PA), Brasil. E-mail: nurse.michelemonteiro@gmail.com. Orcid iD: https://orcid.org/00000003-2239-9721

Endereço para correspondência: Cintia Yolette Urbano Pauxis Aben-Athar. Faculdade de Enfermagem do Instituto de Ciências da Saúde da UFPA. Rua Augusto Correa, 1 - Guamá. Belém (PA), Brasil. CEP 66075-110. E-mail: abenathar_cintia@hotmail.com 


\section{INTRODUÇÃO}

O câncer de mama é caracterizado pelo crescimento descontrolado de células no tecido epitelial mamário, proveniente de alteraçóes genéticas e epigenéticas. Esse câncer é o mais comum entre mulheres, com prevalência crescente após a menopausa ${ }^{1}$. Entre os casos, $5-10 \%$ estáo associados a defeitos genéticos herdados com alta penetrância e $90-95 \%$ relacionados aos fatores ambientais e estilo de vida ${ }^{2}$.

No Brasil, o aumento da sua incidência exigiu respostas do Sistema Nacional de Saúde e, em 2013, foi instituída a Política Nacional para a Prevençáo e Controle do Câncer (PNPCC), na Rede de Atenção à Saúde (RAS), no âmbito do Sistema Único de Saúde (SUS), por meio de açóes de promoção da saúde, prevenção, detecção precoce e tratamento oportuno do câncer, sendo a formação profissional uma diretriz dessa política ${ }^{3}$. As açóes e serviços voltados à detecção precoce e ao tratamento oportuno do câncer de mama estáo inclusos em todos os níveis de atenção à saúde no SUS, com a presença do enfermeiro em todas as açôes ${ }^{4,5}$. No entanto, a organização da rede de atenção ao câncer de mama ainda é um desafio, mostrando-se fragmentada e não garantindo a integralidade do cuidado 6 .

Interessantemente, o câncer hereditário não foi incluso como objeto de cuidado na Política Nacional de Atenção Integral às Pessoas com Doenças Raras no SUS7. Essa política foi a primeira a citar o aconselhamento genético como habilidade da equipe de saúde multiprofissional, requerendo conhecimento sobre conceitos de genética e genômica.

Sinérgico a esses avanços, o Conselho Federal de Enfermagem (Cofen) regulamentou a atuação do enfermeiro no aconselhamento genético de acordo com seu nível de competência técnica (gerais e específicas) ${ }^{8}$ e incluiu a Genética e Biologia Molecular na lista de especialidades ${ }^{9}$. Contudo, nem todas as instituiçóes de ensino superior (IES) acompanharam esse contexto epidemiológico e o avanço da profissão.

As Diretrizes Curriculares Nacionais dos Cursos de Graduação em Enfermagem (DCN/ENF) têm norteado o processo de construção de Projetos Pedagógicos pautados nos preceitos do SUS e na flexibilizaçáo regional conforme as necessidades sociais em saúde ${ }^{10}$, resultando em autonomia didático-científica da grade curricular entre as IES brasileiras.

Sabe-se que essa lacuna na formação profissional pode impactar não somente à pesquisa básica e aplicada ${ }^{11}$, mas principalmente sobre o cuidado. Estudos demonstram um conhecimento limitado sobre genética e câncer de mama entre acadêmicos ${ }^{12}$ e enfermeiros ${ }^{13,14}$, sendo esse profissional importante nas açóes de detecção precoce, no tratamento oportuno e no aconselhamento genético ${ }^{7}$.

Considerando esse contexto, é plausível afirmar que as diferenças nos currículos das IES podem resultar em níveis de conhecimento heterogêneos sobre genética e genômica aplicadas ao câncer de mama entre acadêmicos de enfermagem. Ademais, é importante ressaltar que isso pode reverberar sobre as habilidades práticas desse profissional em todos os níveis de atenção, principalmente na Atenção Primária à Saúde, cuja atuação do enfermeiro pode contribuir na identificação precoce da doença, no gerenciamento de risco e na redução de indicadores de morbimortalidade. Assim, este estudo buscou analisar se o conhecimento dos acadêmicos de enfermagem sobre os conceitos de genética e genômica aplicados ao câncer de mama está associado à grade curricular das instituições onde estudam.

\section{MÉTODO}

Trata-se de um estudo multicêntrico, transversal, norteado pela ferramenta STROBE, que avaliou o conhecimento dos acadêmicos de enfermagem no período de agosto a outubro de 2018.

As IES foram escolhidas por amostragem por conveniência, levando-se em consideração o Projeto Pedagógico do Curso (PPC) disponível no site institucional, bem como a concessáo da carta de anuência institucional à realização do estudo. Foram convidadas sete IES, porém duas declinaram a sua participação, resultando na inclusão de três IES privadas (com a oferta da disciplina genética) e duas públicas (que não a ofertavam) na cidade de Belém, Pará.

A população-alvo foi composta por 10.869 acadêmicos matriculados no curso de graduação em Enfermagem (presencial e a distância) nas IES do Pará no ano de $2017^{15}$. Foi realizada amostragem aleatória simples; adotou-se como frequência esperada 50\% (abordagem apenas dos acadêmicos em aula presencial) e a margem de erro aceitável foi de 5\%. Para o número amostral, foi considerado como limite mínimo para coleta o nível de confiança de 90\%, resultando em 264 participantes; e limite máximo, o nível de confiança de $95 \%$, resultando em 371 amostras. O cálculo amostral foi realizado na função StartCalc do programa Epi Info versão 7.2.2.16 para amostragem aleatória simples conforme as condiçôes citadas anteriormente.

Foram recrutados os alunos que estavam na turma do sexto período, uma vez que a disciplina de genética da grade curricular é ofertada nos semestres iniciais. Assim, foram inclusos os participantes com: (i) idade superior a 18 anos; (ii) ambos os sexos; (iii) regulamente matriculados 
a partir do sexto semestre; e (iv) que aceitaram participar voluntariamente e assinaram o Termo de Consentimento Livre e Esclarecido (TCLE). Os critérios de exclusão foram aqueles que não se enquadraram na elegibilidade dos participantes.

Diante da ausência de questionários que envolvessem as variáveis investigadas neste estudo, criou-se um instrumento de coleta de dados baseado no Consenso Essentials of genetic and genomic nursing: competencies, curricula guidelines, and outcome indicators (Fundamentos da enfermagem genética e genômica: competências, diretrizes curriculares e indicadores de resultados) ${ }^{16}$ e nas políticas de saúde relacionadas ao câncer de mama.

Inicialmente, o questionário foi submetido à estratégia de Validação de Conteúdo por meio da técnica Delphi mais simplificada, sem inferência estatística, seguindo as etapas: seleção dos participantes, elaboração dos questionários, participação e validação de conteúdo por "experts" ou especialistas e análise dos questionários até a obtençáo do nível de consenso ${ }^{17}$. O processo de validação de conteúdo ocorreu em julho de 2018. Foram enviados por e-mail a carta convite, o instrumento de avaliação e o questionário produzido aos especialistas. Cada item do questionário foi avaliado quanto à apresentação, à clareza, à extensão dos enunciados e ao tempo de respostas e julgado da seguinte maneira: concordo, discordo e sugestôes.

Participaram da validação dez enfermeiros acima de 18 anos, atuantes direta ou indiretamente na oncologia, podendo ser docentes, pesquisadores ou residentes do Programa de Residência Multiprofissional do Hospital Universitário João de Barros Barreto da Universidade Federal do Pará. Todas as sugestôes foram acatadas.

Em seguida, realizou-se um estudo-piloto com cinco acadêmicos de enfermagem para ajustes do instrumento diante das dificuldades de compreensão das questôes, coerência e média de tempo necessário para o seu preenchimento. Tal atividade garantiu o aprimoramento do questionário, com a eliminação dos elementos confundidores presentes durante a sua testagem.

A versão definitiva do questionário foi composta de 19 questóes referentes a três domínios (caracterização sociodemográfica, conceitos básicos de genética e genômica e câncer de mama). As perguntas foram dicotômicas (verdadeira ou falsa) ou de múltipla escolha. Os dados do teste-piloto não foram inclusos no resultado.

Para a coleta de dados, todos os pesquisadores foram treinados para que a aplicação do questionário fosse a mais uniforme possível. Os objetivos do projeto foram apresentados aos participantes em uma abordagem individual e isolada, a fim de preservar a sua privacidade, evitar constrangimento e assegurar a participaçáo voluntária.
Após o aceite e a assinatura do TCLE, os autores entregavam o instrumento e depois se retiravam. A conclusão de preenchimento dos instrumentos demorou em média 15 minutos. Uma vez preenchidos, os questionários foram colocados em envelope sem identificação e entregues ao pesquisador.

A principal hipótese a ser testada foi analisar se o conhecimento dos acadêmicos de enfermagem sobre os conceitos de genética e genômica aplicados ao câncer de mama está associado ao tipo de IES (pública $v s$. privada) onde estudam. A hipótese secundária testada foi se as características dos acadêmicos associam-se às IES onde estudavam.

Para testar a hipótese, as variáveis incluídas foram de natureza categórica. Na primeira hipótese, considerou-se a variável preditora (tipo de IES); enquanto as variáveis de resposta foram as questôes sobre conhecimentos básicos sobre genética e genômica (conceito de DNA, conceito de gene, conceito de genética, conceito de cromossomo, conceito de mutaçóes), conhecimentos sobre câncer (conceito esporádico ou hereditário) e conhecimentos sobre o câncer de mama (exame clínico, rastreamento mamográfico e sua periodicidade entre mulheres sob risco geral ou alto risco).

Alternativamente, para testar a hipótese secundária, as variáveis preditoras foram: faixa etária; gênero; cidade; situação ocupacional; e ter cursado disciplina genética na graduação. Enquanto a variável de resposta foi o conhecimento referente ao tipo de IES (pública vs. privada).

Os participantes receberam um código alfanumérico aleatório conforme sua ordem de abordagem para preservação do anonimato. Os dados foram digitados em um banco de dados do programa software Epi Info versão 7.2.2.16 e a análise descritiva para caracterizar a população do estudo foi realizada no programa Microsof Excel ${ }^{\circ}$.

As variáveis categóricas foram calculadas pelas frequências absolutas e relativas. Para a variável contínua (idade), foi realizada uma análise exploratória no programa Minitab $14^{\oplus}$. Considerando a natureza categórica das variáveis, foram aplicados o teste qui-quadrado de Person e o teste $G$, sendo que neste último as variáveis com frequências esperadas eram menores do que cinco. Foi adotado nível de significância de $\mathrm{p}<0,05$ no programa Bioestat $5.3^{\circ}$.

Este estudo atendeu a todas as exigências das Resoluçóes do Conselho Nacional de Saúde no ${ }^{\circ} .466 / 2012^{18}$ e n ${ }^{\circ}$. 510/2016 $6^{19}$, com parecer favorável (1.593.095) do Comitê de Ética em Pesquisa (CAAE 56366216.1.3001.0018).

\section{RESULTADOS}

Aproximadamente, 323 estudantes de enfermagem com idade média de $27,4 \pm 6,6$, a maioria do sexo feminino 
$(80,18 \% \mathrm{n}=259 / 323)$, da Regiáo Metropolitana de Belém $(96,28 \%, \mathrm{n}=311 / 323)$, que só estudavam $(59,46 \%$, $\mathrm{n}=64 / 323)$ e que foram recrutados para o estudo. Cerca de $80,18 \%$, ( $\mathrm{n}=259 / 323)$ matriculados em universidades particulares e 19,82\% ( $n=64 / 323)$ em universidades públicas (Tabela 1).

As IES avaliadas mostraram divergência no PPC. Por conseguinte, os acadêmicos de enfermagem das IES privadas afirmaram que a disciplina de genética foi ofertada, enquanto nas públicas não $(\mathrm{p}=0,0001)$. O primeiro contato dos alunos das IES privadas ocorreu durante a graduação e, nas faculdades públicas, esse acesso foi variável (graduação, cursos, entre outros) ( $\mathrm{p}=0,0032)$. A Tabela 2 mostra associação estatisticamente significativa entre ser acadêmico de IES pública e não conhecer os conceitos básicos de genética e genômica $(0,0045)$. As demais variáveis não apresentaram associação à IES.

A análise dos resultados do conhecimento sobre câncer de mama encontrou associaçáo estatisticamente significante entre ser acadêmico de IES privada e não conhecer a periodicidade da recomendação do exame clínico da mama anualmente a partir dos 40 anos $(\mathrm{p}=0,0009)$. Adicionalmente, o mesmo grupo náo reconheceu a periodicidade do rastreamento mamográfico na população geral $(0,0021)$. Por outro lado, a maioria dos participantes nos dois grupos acertou a faixa etária indicada para o rastreamento mamográfico para a população sob alto risco (familiar) $(0,0431)$, conforme a Tabela 3 .

\section{DISCUSSÃO}

Este estudo avaliou o conhecimento dos acadêmicos de enfermagem no câncer de mama sobre os conceitos básicos de genética e genômica, fatores de risco e rastreamento. Identificou-se que a maioria dos participantes foi mulheres, com faixa etária entre 21 e 30 anos, residentes na Região Metropolitana de Belém ( $\mathrm{p}=0,021)$, com dedicação exclusiva aos estudos nas IES públicas e rotina de estudo e/ou trabalho nas IES privadas.

Há uma relativa escassez de publicaçóes sobre a avaliação do conhecimento de acadêmicos de enfermagem brasileiros sobre genética e genômica e a avaliação desse conhecimento aplicado ao câncer de mama ainda é inexistente. Os resultados deste estudo revelaram um perfil sociodemográfico semelhante ao estudo de Munroe e Loerzel ${ }^{12}$.

$\mathrm{O}$ presente estudo mostrou que os acadêmicos de enfermagem das IES privadas afirmaram ter cursado a disciplina, enquanto os acadêmicos das instituiçóes públicas a negaram $(\mathrm{p}=0,0001)$. Assim, o primeiro contato dos alunos das IES privadas ocorreu durante a graduação e, no outro grupo, esse acesso variou na graduação, cursos, ou outros $(\mathrm{p}=0,0032)$.

A divergência na formação do acadêmico ocorre com a flexibilização das DCN/ENF, ditadas por políticas de saúde, mercado de trabalho e regionalismo. A abordagem genética não é citada diretamente, mas sim sub-representada na base curricular de ciências biológicas e da saúde ${ }^{10}$, sendo ministrada nos semestres iniciais.

Tabela 1. Distribuição dos entrevistados de acordo com o perfil sociodemográfico por Instituição de Ensino Superior Privada e Pública, Belém, Pará, Brasil, 2018

\begin{tabular}{|c|c|c|c|c|c|c|}
\hline \multicolumn{2}{|c|}{ Variáveis } & \multirow{2}{*}{$\begin{array}{c}\text { Privada } \\
\text { N (259) } \\
50\end{array}$} & \multirow{2}{*}{$\begin{array}{c}\% \\
19,3\end{array}$} & \multirow{2}{*}{$\begin{array}{c}\text { Pública } \\
\text { N (64) } \\
14\end{array}$} & \multirow{2}{*}{$\begin{array}{l}\% \\
21,9\end{array}$} & \multirow{2}{*}{$\begin{array}{c}\mathbf{p} \\
0,7743^{a}\end{array}$} \\
\hline Gênaro & Masculino & & & & & \\
\hline Genero & Feminino & 209 & 80,7 & 50 & 78,1 & \\
\hline \multirow{6}{*}{ Idade } & $\leq 20$ & 13 & 5,0 & 2 & 3,1 & $1,000^{b}$ \\
\hline & 21 a 30 & 193 & 74,5 & 58 & 90,6 & \\
\hline & 31 a 40 & 41 & 15,8 & 5 & 7,8 & \\
\hline & 41 a 50 & 11 & 4,2 & 1 & 1,6 & \\
\hline & 51 a 60 & 1 & 0,4 & 0 & 0,0 & \\
\hline & $>60$ & 0 & 0,0 & 0 & 0,0 & \\
\hline \multirow{3}{*}{ Cidade } & Região Metropolitana & 254 & 98,1 & 57 & 89,1 & $0,021^{b}$ \\
\hline & Outras Regiões & 5 & 1,9 & 6 & 9,4 & \\
\hline & Não informado & 0 & 0,0 & 1 & 1,6 & \\
\hline \multirow{2}{*}{ Situação ocupacional } & Trabalha e estuda & 105 & 40,54 & 0 & 0 & $0,698^{b}$ \\
\hline & Apenas estuda & 154 & 59,46 & 64 & 100 & \\
\hline
\end{tabular}

(a) Teste do qui-quadrado.

(b) Teste G. 
Tabela 2. Associação de conhecimentos sobre genética e genômica entre as Instituições de Ensino Superior, Belém, Pará, Brasil, 2018

\begin{tabular}{|c|c|c|c|c|c|c|}
\hline Variáveis & & $\begin{array}{l}\text { Privada } \\
\text { N (259) }\end{array}$ & $\%$ & $\begin{array}{l}\text { Pública } \\
\text { N (64) }\end{array}$ & $\%$ & $\mathbf{p}$ \\
\hline \multirow{5}{*}{$\begin{array}{l}\text { Houve a disciplina } \\
\text { genética na sua } \\
\text { graduação? }\end{array}$} & $\operatorname{Sim}$ & 189 & 73,0 & 5 & 7,8 & $<0,0001^{b}$ \\
\hline & Não & 70 & 27,0 & 59 & 92,2 & \\
\hline & Graduação & 170 & 65,6 & 36 & 56,3 & $0,0032^{b}$ \\
\hline & Curso & 10 & 3,9 & 9 & 14,1 & \\
\hline & Estágio extracurricular & 0 & 0,0 & 0 & 0,0 & \\
\hline \multirow{7}{*}{$\begin{array}{l}\text { Qual seu primeiro } \\
\text { contato com a } \\
\text { genética/genômica? }\end{array}$} & Especialização & 0 & 0,0 & 0 & 0,0 & \\
\hline & Mestrado & 0 & 0,0 & 0 & 0,0 & \\
\hline & Doutorado & 0 & 0,0 & 0 & 0,0 & \\
\hline & Outros & 14 & 5,4 & 9 & 14,1 & \\
\hline & Não Respondeu & 0 & 0,0 & 6 & 9,4 & \\
\hline & Nenhum contato & 68 & 26,3 & 1 & 1,6 & \\
\hline & Acertos & 208 & 80,3 & 56 & 87,5 & $0,0952^{a}$ \\
\hline \multirow[t]{3}{*}{ Conceito de DNA } & Erros & 51 & 19,7 & 6 & 9,4 & \\
\hline & Sem resposta & 0 & 0,0 & 2 & 3,1 & \\
\hline & Acertos & 222 & 85,7 & 51 & 79,7 & $0,4543^{a}$ \\
\hline \multirow[t]{3}{*}{ Conceito de gene } & Erros & 37 & 14,3 & 12 & 18,8 & \\
\hline & Sem resposta & 0 & 0,0 & 1 & 1,6 & \\
\hline & Acertos & 204 & 78,8 & 37 & 57,8 & $0,0045^{a}$ \\
\hline \multirow{3}{*}{$\begin{array}{l}\text { Conceito de genética/ } \\
\text { genômica }\end{array}$} & Erros & 54 & 20,8 & 24 & 37,5 & \\
\hline & Sem resposta & 1 & 0,4 & 3 & 4,7 & \\
\hline & Acertos & 229 & 88,4 & 57 & 89,1 & $0,941^{a}$ \\
\hline \multirow{3}{*}{$\begin{array}{l}\text { Conceito de } \\
\text { cromossomo }\end{array}$} & Erros & 30 & 11,6 & 7 & 10,9 & \\
\hline & Sem resposta & 0 & 0,0 & 0 & 0,0 & \\
\hline & Acertos & 240 & 92,7 & 57 & 89,1 & $0,7493^{a}$ \\
\hline \multirow[t]{2}{*}{ Conceito de mutações } & Erros & 19 & 7,3 & 6 & 9,4 & \\
\hline & Sem resposta & 0 & 0,0 & 1 & 1,6 & \\
\hline
\end{tabular}

(a) Teste do qui-quadrado.

(b) Teste G.

Aqui, identificou-se um alto números de acertos sobre os conceitos de genética e genômica entre os acadêmicos de enfermagem, com maior destaque da IES privada em detrimento à pública $(\mathrm{p}=0.0045)$. Embora um estudo tenha revelado resultados semelhantes sobre o reconhecimento da aplicabilidade clínica desses conceitos ${ }^{12}$ pelos acadêmicos, há possibilidade de que esse conhecimento seja residual do segundo grau ${ }^{20}$.

Além do mais, a distribuição da carga horária teórica e prática depende da teoria de aprendizagem adotada pela instituição ${ }^{21}$. Segundo os PPC das IES públicas, há maior valorização da prática em campo, vivência e reflexão do acadêmico quanto aos cuidados prestados para posterior correlação com a fundamentação teórica. Com isso, pode-se inferir que a carga horária teórica é maior e mais conteudista na IES privada, justificando-se as assertivas mais acentuadas pela vivência da disciplina genética.

É válido ressaltar que as lacunas de conhecimento sobre os conceitos básicos de genética e genômica tendem a impactar negativamente a formação profissional, a subutilização da força de trabalho e as melhorias as condiçốes de saúde à população ${ }^{16,14}$. Para tanto, mudanças no currículo brasileiro são essenciais para acompanhar o cenário epidemiológico do câncer quanto à incidência e à morbimortalidade, bem como a evoluçáo da profissão na assistência complexa para essa paciente.

O câncer de mama é considerado um problema de saúde pública e, para isso, é essencial o estabelecimento de controle de fatores de risco e rastreamento adequado, bem como profissionais capacitados ${ }^{4,5}$. Neste estudo, a 
Tabela 3. Distribuição dos acadêmicos de enfermagem entre as Instituições de Ensino Superior de acordo com conhecimentos sobre o câncer de mama, Belém, Pará, Brasil, 2018

\begin{tabular}{|c|c|c|c|c|c|c|}
\hline \multirow{2}{*}{ Variáveis } & & \multicolumn{2}{|l|}{ Privadas } & \multicolumn{2}{|c|}{ Públicas } & \multirow{2}{*}{$\mathbf{p}$} \\
\hline & & $N(259)$ & $\%$ & $N(64)$ & $\%$ & \\
\hline \multirow{3}{*}{$\begin{array}{l}\text { A maioria dos casos de } \\
\text { câncer de mama ocorre } \\
\text { em razão de alterações } \\
\text { genéticas hereditárias? }\end{array}$} & Acertos & 204 & 78,76 & 44 & 68,75 & \multirow{3}{*}{$0,5621^{\mathrm{a}}$} \\
\hline & Erros & 54 & 20,85 & 15 & 23,44 & \\
\hline & Não informado & 01 & 0,39 & 05 & 7,81 & \\
\hline \multirow{3}{*}{$\begin{array}{l}\text { A maioria dos casos de } \\
\text { câncer de mama ocorre } \\
\text { por causa de alterações } \\
\text { genéticas associadas aos } \\
\text { fatores ambientais? }\end{array}$} & Acertos & 150 & 57,92 & 29 & 45,31 & \multirow{3}{*}{$0,1852^{a}$} \\
\hline & Erros & 109 & 42,08 & 32 & 50,00 & \\
\hline & Não informado & 00 & 0,00 & 03 & 4,69 & \\
\hline \multirow{3}{*}{$\begin{array}{l}\text { Menarca precoce, } \\
\text { menopausa tardia, } \\
\text { nuliparidade, alterações } \\
\text { hormonais, sedentarismo, } \\
\text { sobrepeso, tabagismo e } \\
\text { terapia hormonal são os } \\
\text { principais fatores de risco } \\
\text { para o câncer de mama? }\end{array}$} & Acertos & 241 & 93,05 & 57 & 89,06 & \multirow[b]{3}{*}{$0,9748^{b}$} \\
\hline & Erros & 18 & 6,95 & 05 & 7,81 & \\
\hline & Não informado & 00 & 0,00 & 02 & 3,13 & \\
\hline \multirow{3}{*}{$\begin{array}{l}\text { Câncer de mama } \\
\text { diagnosticados em homens } \\
\text { aumenta o risco de } \\
\text { desenvolver câncer de mama } \\
\text { hereditário? }\end{array}$} & Acertos & 205 & 79,15 & 42 & 65,63 & \multirow{3}{*}{$0,1750^{a}$} \\
\hline & Erros & 54 & 20,85 & 18 & 28,13 & \\
\hline & Não informado & 00 & 0,00 & 04 & 6,25 & \\
\hline \multirow{3}{*}{$\begin{array}{l}\text { O exame clínico deve ser } \\
\text { realizado em pacientes, } \\
\text { anualmente, a partir de } 40 \\
\text { anos? }\end{array}$} & Acertos & 77 & 29,73 & 32 & 50,00 & \multirow{3}{*}{$0,0009^{a}$} \\
\hline & Erros & 182 & 70,27 & 28 & 43,75 & \\
\hline & Não informado & 00 & 0,00 & 04 & 6,25 & \\
\hline \multirow{4}{*}{$\begin{array}{l}\text { Qual é a faixa etária } \\
\text { indicada para o } \\
\text { rastreamento mamográfico à } \\
\text { população geral? }\end{array}$} & 35- 69 anos & 148 & 57,14 & 44 & 68,75 & \multirow{4}{*}{$0,1138^{a}$} \\
\hline & 50- 69 anos & 108 & 41,70 & 18 & 28,13 & \\
\hline & $60-79$ anos & 03 & 1,16 & 00 & 0,00 & \\
\hline & Não informado & 00 & 0,00 & 02 & 3,13 & \\
\hline \multirow{5}{*}{$\begin{array}{l}\text { Qual é a faixa etária } \\
\text { indicada para o } \\
\text { rastreamento mamográfico } \\
\text { à população com risco } \\
\text { familiar? }\end{array}$} & 35- 69 anos & 202 & 77,99 & 54 & 84,38 & \multirow{4}{*}{$0,0431^{b}$} \\
\hline & 50- 69 anos & 46 & 17,76 & 07 & 10,94 & \\
\hline & $60-79$ anos & 11 & 4,25 & 00 & 0,00 & \\
\hline & Não informado & 00 & 0,00 & 03 & 4,69 & \\
\hline & 1 ano & 143 & 55,21 & 48 & 75,00 & \multirow{4}{*}{$0,0021^{a}$} \\
\hline \multirow{3}{*}{$\begin{array}{l}\text { Qual a periodicidade do } \\
\text { rastreamento mamográfico } \\
\text { na população geral? }\end{array}$} & 2 anos & 83 & 32,05 & 07 & 10,94 & \\
\hline & 3 anos & 33 & 12,74 & 06 & 9,38 & \\
\hline & Não informado & 00 & 0,00 & 03 & 4,69 & \\
\hline
\end{tabular}

(a) Teste do qui-quadrado.

(b) Teste G.

maioria dos acadêmicos da IES privada equivocou-se na recomendação do exame clínico da mama a partir dos 40 anos $(\mathrm{p}=0,00009)$.

O Ministério da Saúde aconselha o exame clínico das mamas (ECM) como um método de rastreamento padronizado, sistemático e com periodicidade fixa (anual) a partir dos 40 anos. Este deve ser realizado pelo médico ou enfermeiro(a) durante a consulta na atenção primária e direciona a mamografia em caso de necessidade ${ }^{22}$.

A discussão dos conceitos básicos de genética e gnômica aplicados ao câncer de mama na graduação permite entender a diferença entre doença esporádica 
e hereditária, assim como subsidia a orientação sobre o intervalo adequado de rastreio do câncer de mama conforme o tipo de risco ao qual o indivíduo esteja susceptível ${ }^{22,14}$. Entretanto, apesar do grande apelo epidemiológico, a disciplina sobre o câncer (oncologia) ainda não é realidade nas IES de enfermagem no Estado do Pará. Interessantemente, a doença também se mostra subrepresentada na mesma base curricular que a genética "base molecular e celular nos processos normais e alterados" ou no conteúdo curricular "assistência em enfermagem"

Outro erro cometido pelos acadêmicos da IES privada foi relacionado à periodicidade bienal do rastreamento mamográfico à população geral $(\mathrm{p}=0,0021)$. Alternativamente, os dois grupos apresentaram alto número de acertos quanto à faixa etária alvo recomendada para o rastreamento mamográfico tanto para a população sob risco relativo $(\mathrm{p}=0,0021)$ quanto para o alto risco $(\mathrm{p}=0,0431)$. A mamografia bienal entre 50 e 69 anos é a única estratégia de rastreamento reconhecida para o câncer esporádico ${ }^{22}$.

Este foi o primeiro estudo a avaliar o conhecimento de acadêmicos de enfermagem sobre a periodicidade da mamografia conforme a estratificação do risco geral ou familiar. Acredita-se que uma maior prática curricular em serviços vinculados ao SUS permitirá maior vivência das políticas de saúde, voltando os acadêmicos às açôes de detecção precoce e ao tratamento oportuno do câncer de mama, desde que supervisionados pelo enfermeiro, que é seu executor ${ }^{4,5}$.

Sáo citadas como limitaçóes do estudo: (i) a flexibilidade curricular que permite a ausência ou sub-representação do conteúdo sobre genética e genômica e/ou oncologia nos PPC de cada IES; (ii) o número de participantes das universidades públicas; (iii) uma amostragem regional (Estado do Pará), fato que não permite a generalização dos resultados ao restante do Brasil; (iv) a escassez de estudos sobre a temática disponível para a comparação da realidade nacional.

\section{CONCLUSÃO}

Em conclusão, a maioria dos acadêmicos de enfermagem era mulheres, dedicadas apenas à graduação, com média de idade de $27,4 \pm 6,6$, provenientes da Região Metropolitana de Belém e de instituição privada. Os resultados mostraram que os acadêmicos de IES privada apresentaram maiores acertos sobre conceitos de genética e genômica, pois continham a disciplina genética na grade curricular, enquanto os acadêmicos da IES pública se destacaram nos acertos relacionados ao câncer de mama sobre políticas de saúde, em razão da maior vivência prática no estágio curricular.
Sabe-se que as lacunas encontradas na formação profissional podem limitar a atuação do enfermeiro na pesquisa básica e aplicada, na detecção precoce rastreamento e no tratamento oportuno ao câncer de mama, bem como na educação em saúde que permeia todas as etapas anteriores. Alarmantemente, o apelo epidemiológico (alta morbimortalidade da doença) e o reconhecimento da atuação profissional por entidades de classe ainda não foram suficientes para adaptação às DCN/ENF.

Finalmente, espera-se que os resultados deste estudo possam gerar reflexóes quanto à reconfiguração curricular para as IES estaduais, diante da necessidade de máo de obra qualificada local. Para tanto, sugerem-se estudos adicionais sobre a temática com benefícios diretos ao ensino, pesquisa e extensão.

\section{CONTRIBUIÇÕES}

Aline Maria Pereira Cruz Ramos contribuiu na concepção e/ou no planejamento do estudo; na obtenção, análise e interpretação dos dados; assim como na redação e revisão crítica. Ingrid Renny Silva Palha; Cintia Yolette Urbano Pauxis Aben-Athar; Karolayne Teles Costa; Renata Glaucia Barros da Silva Lopes; Michele Monteiro Sousa; e Glenda Roberta Oliveira Naiff Ferreira participaram da obtenção, análise e interpretação dos dados; assim como da redação e revisão crítica. Todos os autores aprovaram a versão final a ser publicada.

\section{DECLARAÇÃO DE CONFLITO DE INTERESSES}

Nada a declarar.

\section{FONTES DE FINANCIAMENTO}

Não há.

\section{REFERÊNCIAS}

1. Dumitrescu RG. Interplay between genetic and epigenetic changes in breast cancer subtypes. Methods Mol Biol. 2018;1856:19-34. doi: https://doi.org/10.1007/978-14939-8751-1_2

2. Low SK, Zembutsu H, Nakamura Y. Breast cancer: the translation of big genomic data to cancer precision medicine. Cancer Sci. 2018;109(3):497-506. doi: https://doi.org/10.1111/cas.13463

3. Ministério da Saúde (BR), Secretaria de Atenção à Saúde, Departamento de Atenção Básica. Controle dos cânceres do colo do útero e da mama [Internet]. 2. ed. Brasília, DF: Ministério da Saúde; 2013 [acesso 2020 out 22]. (Cadernos de Atenção Básica; no. 13). Disponível em: 
http://bvsms.saude.gov.br/bvs/publicacoes/controle_ canceres_colo_utero_2013.pdf

4. Ministério da Saúde (BR). Protocolos da atenção básica: saúde das mulheres [Internet]. Brasília, DF: Ministério da Saúde, Instituto Sírio-Libanês de Ensino e Pesquisa; 2016 [acesso 2020 out 22]. Disponível em: http://bvsms. saude.gov.br/bvs/publicacoes/protocolos_atencao_ basica_saude_mulheres.pdf

5. Ministério da Saúde (BR). Portaria conjunta $n^{\circ} 19$, de 3 de julho de 2018. Aprova as Diretrizes Diagnósticas e Terapêuticas do Carcinoma de Mama [Internet]. Diário Oficial da União. 2018 jul 16 [acesso 2020 out 22]; Edição 135, Seção 1:59. Disponível em: https://www. in.gov.br/materia/-/asset_publisher/Kujrw0TZC2Mb/ content/id/31547238

6. Goldman RE, Figueiredo EN, Fustinoni SM, et al. Brazilian breast cancer care network: the perspective of health managers. Rev Bras Enferm. 2019;72(Suppl 1):274-81. doi: https://doi.org/10.1590/0034-71672017-0479

7. Ministério da Saúde (BR). Portaria nº 199 de 30 de janeiro de 2014. Institui a Política Nacional de Atenção Integral às Pessoas com Doenças Raras, aprova as Diretrizes para Atenção Integral às Pessoas com Doenças Raras no âmbito do Sistema Único de Saúde (SUS) e institui incentivos financeiros de custeio [Internet]. Diário Oficial da União. 2014 fev 12 [acesso 2021 jan 16]; Seção 1:44. Disponível em: https://bvsms.saude.gov. br/bvs/saudelegis/gm/2014/prt0199_30_01_2014.html

8. Conselho Federal de Enfermagem (BR). Resolução $n^{\circ}$ 468, de 17 de dezembro de 2014. Normatiza a atuação do Enfermeiro em Aconselhamento Genético [Internet]. Diário Oficial da União. 2015 jan 22 [acesso 2020 out 22]; Edição 15, Seção 1:103. Disponível em: https://www. in.gov.br/materia/-/asset_publisher/Kujrw0TZC2Mb/ content/id/32122359/do1-2015-01-22-resolucao-n468-de-17-de-dezembro-de-2014-32122187

9. Conselho Federal de Enfermagem (BR). Decisão $n^{\circ}$ 245, de 27 de outubro de 2016. Resoluçáo Cofen no 389/2011. Registro de Especialidade. Genética e Biologia Molecular. [Internet]. Diário Oficial da União. 2016 nov 1 [acesso 2019 out 22]; Edição 210, Seção 1:110. Disponível em: http:/www.cofen.gov.br/wp-content/ uploads/2016/10/Dec-245-16.pdf

10. Conselho Nacional de Saúde (BR). Resolução no 573, de 31 de janeiro de 2018. Aprova o Parecer Técnico no 28/2018 contendo recomendaçóes do Conselho Nacional de Saúde (CNS) à proposta de Diretrizes Curriculares Nacionais (DCN) para o curso de graduaçáo Bacharelado em Enfermagem, conforme anexo [Internet]. Diário Oficial da União. 2018 nov 6 [acesso 2021 jan 16]; Edição 213, Seção 1:38. Disponível em: https://www.in.gov.br/ materia/-/asset_publisher/Kujrw0TZC2Mb/content/ id/48743098/do1-2018-11-06-resolucao-n-573-de-31-de
11. Barbato ES, Daly BJ, Darrah RJ. Educating nursing scientists: integrating genetics and genomics into $\mathrm{PhD}$ curricula. J Prof Nurs. 2019;35(2)89-92. doi: https:// doi.org/10.1016/j.profnurs.2018.09.002

12. Munroe T, Loerzel V. Assessing nursing students' knowledge of genomic concepts and readiness for use in practice. Nurse Educ. 2016;41(2)86-9. doi: https://doi. org/10.1097/NNE.0000000000000210

13. Pruitt LCC, Odedina S, Anetor I, et al. Breast cancer knowledge assessment of health workers in Ibadan, southwest Nigeria. JCO Glob Oncol. 2020;6:387-94. doi: https://doi.org/10.1200/JGO.19.00260

14. Prolla CMD, Silva PS, Oliveira Netto CB, et al. Conhecimento sobre câncer de mama e câncer de mama hereditário entre enfermeiros em um hospital público. Rev Latino-Am Enferm. 2015;23(1):90-7. doi: https:// doi.org/10.1590/0104-1169.0185.2529

15. Instituto Nacional de Estudos e Pesquisas Educacionais Anísio Teixeira. Sinopses estatísticas da educação superior 2017 [Internet]. Brasília, DF: INEP; 2017 [acesso 2020 out 22]. Disponível em: http://inep.gov.br/web/guest/ sinopses-estatisticas-da-educacao-superior

16. Calzone KA, Jenkins J, Prows CA, et al. Establishing the outcome indicators for the Essential Nursing Competencies and Curricula Guidelines for Genetics and Genomics. J Prof Nurs. 2011;27(3):179-91. doi: https:// doi.org/10.1016/j.profnurs.2011.01.001

17. Perroca MG. Desenvolvimento e validação de conteúdo da nova versão de um instrumento para classificação de pacientes. Rev Latino-Am Enfermagem [Internet]. 2011 [acesso 2020 out 22];19(1). Disponível em: https://www. scielo.br/pdf/rlae/v19n1/pt_09.pdf

18. Conselho Nacional de Saúde (BR). Resolução no 466, de 12 de dezembro de 2012. Aprova as diretrizes e normas regulamentadoras de pesquisas envolvendo seres humanos [Internet]. Diário Oficial da União. 2013 jun 13 [acesso 2020 out 22]; Seção 1:59. Disponível em: http://conselho. saude.gov.br/resolucoes/2012/Reso466.pdf

19. Conselho Nacional de Saúde (BR). Resolução no 510 , de 7 de abril de 2016. Dispóe sobre as normas aplicáveis a pesquisas em Ciências Humanas e Sociais cujos procedimentos metodológicos envolvam a utilização de dados diretamente obtidos com os participantes ou de informaçóes identificáveis ou que possam acarretar riscos maiores do que os existentes na vida cotidiana, na forma definida nesta Resoluçáo [Internet]. Diário Oficial da União. 2016 maio 24 [acesso 2020 out 22]; Edição 98, Seção 1:44. Disponível em: https://www.in.gov.br/ materia/-/asset_publisher/Kujrw0TZC2Mb/content/ $\mathrm{id} / 22917581$

20. Bashore LM, Daniels G, Borchers L, et al. Facilitating faculty competency to integrate genomics into nursing curriculum within a private US University. Nurs Res. 2018;8:9-14. doi: https://doi.org/10.2147/NRR. S165852 
21. Sousa ATO, Formiga NS, Oliveira SHS, et al. A utilização da teoria da aprendizagem significativa no ensino da enfermagem. Rev Bras Enferm. 2015;68(4):713-21. doi: https://doi.org/10.1590/0034-7167.2015680420i

22. Instituto Nacional de Câncer José Alencar Gomes da Silva. Estimativa 2020: diretrizes para a detecção precoce do câncer de mama no Brasil [Internet]. Rio de Janeiro: INCA; 2019 [acesso 2020 out 22]. Disponível em: https://www.inca.gov.br/sites/ufu.sti.inca.local/files/ media/document/estimativa-2020-incidencia-de-cancerno-brasil.pdf 